\title{
Limits on the Position Wander of Sgr A*
}

\section{Citation}

Reid, Mark J., Avery E. Broderick, Abraham Loeb, Mareki Honma, and Andreas Brunthaler. 2008. "Limits on the Position Wander of Sgr A*." The Astrophysical Journal 682 (2): 1041-46. https:// doi.org/10.1086/588603.

\section{Permanent link}

http://nrs.harvard.edu/urn-3:HUL.InstRepos:41393459

\section{Terms of Use}

This article was downloaded from Harvard University's DASH repository, and is made available under the terms and conditions applicable to Other Posted Material, as set forth at http:// nrs.harvard.edu/urn-3:HUL.InstRepos:dash.current.terms-of-use\#LAA

\section{Share Your Story}

The Harvard community has made this article openly available.

Please share how this access benefits you. Submit a story.

\section{Accessibility}


The Astrophysical Journal, 682:1041-1046, 2008 August 1

(C) 2008. The American Astronomical Society. All rights reserved. Printed in U.S.A.

\title{
LIMITS ON THE POSITION WANDER OF SGR A*
}

\author{
Mark J. Reid, ${ }^{1}$ Avery E. Broderick, ${ }^{1,2}$ Abraham Loeb, ${ }^{1}$ Mareki Honma, ${ }^{3}$ and Andreas Brunthaler ${ }^{4}$ \\ Received 2008 January 24; accepted 2008 March 27
}

\begin{abstract}
We present measurements with the Very Long Baseline Array of the variability in the centroid position of Sgr A* relative to a background quasar at $7 \mathrm{~mm}$ wavelength. We find an average centroid wander of $71 \pm 45 \mu$ as for timescales between 50 and 100 minutes and $113 \pm 50 \mu$ as for timescales between 100 and 200 minutes, with no secular trend. These are sufficient to begin constraining the viability of the accretion hot spot model for the radio variability of Sgr A*. It is possible to rule out hot spots with orbital radii above $15 G M_{\text {Sgr A* }} / c^{2}$ that contribute more than $30 \%$ of the total $7 \mathrm{~mm}$ flux. However, closer or less luminous hot spots remain unconstrained. Since the fractional variability of Sgr A* during our observations was $\sim 20 \%$ on timescales of hours, the hot spot model for Sgr A*'s radio variability remains consistent with these limits. Improved monitoring of $\mathrm{Sgr} \mathrm{A}^{*}$ 's centroid position has the potential to place significant constraints on the existence and morphology of inhomogeneities in a supermassive black hole accretion flow.
\end{abstract}

Subject headings: accretion, accretion disks — astrometry — black hole physics — Galaxy: center — gravitational lensing — instrumentation: high angular resolution

\section{INTRODUCTION}

There is now overwhelming evidence that $\mathrm{Sgr} \mathrm{A}^{*}$ is a supermassive black hole (SMBH) at the center of the Milky Way. Many stars are observed to orbit about a common focal position, requiring an unseen mass of $\approx 4 \times 10^{6} M_{\odot}$ contained within a radius of less than $100 \mathrm{AU}$ (Schödel et al. 2002; Ghez et al. 2003), for a distance to the center of $8.0 \mathrm{kpc}$ (Reid 1993). Accurate registration of the infrared and radio reference frames (Menten et al. 1997; Reid et al. 2003) reveal that the common orbital focal position is coincident with $\mathrm{Sgr} \mathrm{A}^{*}$ to within measurement uncertainty of $\approx 10$ mas. Finally, the absence of intrinsic motion of Sgr $A^{*}$ at levels near that expected for a $4 \times 10^{6} M_{\odot}$ object (Reid \& Brunthaler 2004), coupled with a size less than 1 AU (see, e.g., Bower et al. 2004), provides a lower limit on mass density of $\sim 10^{22} M_{\odot} \mathrm{pc}^{-3}$, which is only 2 orders of magnitude less than the density of a $4 \times 10^{6} M_{\odot}$ nonrotating black hole within its innermost stable orbit. There can now be little doubt that Sgr A $^{*}$ is a $\mathrm{SMBH}$.

Reid \& Brunthaler (2004) present measurements of the position of $\mathrm{Sgr} \mathrm{A}^{*}$ relative to a compact extragalactic radio source (J1745-2820, also referred to as J1745-283 in earlier publications). These measurements were conducted with the NRAO ${ }^{5}$ Very Long Baseline Array (VLBA) over a period of $8 \mathrm{yr}$ at a wavelength of $7 \mathrm{~mm}(43 \mathrm{GHz})$ and have been used to determine the apparent proper motion of Sgr A*. Over timescales of months or longer, Sgr A*'s apparent motion is dominated by the effects of the orbit of the Sun about the center of the Galaxy. The component of the Sun's orbit in the Galactic plane is uncertain at roughly the $10 \%$ level, and this limits estimation of any intrinsic motion of Sgr $\mathrm{A}^{*}$ at about the $\pm 20 \mathrm{~km} \mathrm{~s}^{-1}$ level. However, the

\footnotetext{
1 Harvard-Smithsonian Center for Astrophysics, 60 Garden Street, Cambridge, MA 02138.

${ }_{2}^{2}$ Canadian Institute for Theoretical Astrophysics, 60 St. George Street, Toronto, ON, Canada.

3 Mizusawa VERA Observatory, NAOJ, Mitaka, Tokyo 181-8588, Japan.

4 Max-Planck-Institute für Radioastronomie, 69 Auf dem Hügel, Bonn53121, Germany.

5 The National Radio Astronomy Observatory is operated by Associated Universities, Inc., under a cooperative agreement with the National Science Foundation.
}

component of the motion of the Sun out of the Galactic plane is known to high accuracy $\left(7.16 \pm 0.38 \mathrm{~km} \mathrm{~s}^{-1}\right.$ toward the north Galactic pole; Dehnen \& Binney 1998). After removing the effects of the Sun's motion, the residual motion of Sgr A* perpendicular to the Galactic plane is very small, $\$ 1 \mathrm{~km} \mathrm{~s}^{-1}$, as expected for a SMBH at the dynamical center of the Galaxy. While previous work concentrated on the long-term motion of Sgr A*, here we analyze its short-term position "wander" on timescales of hours to weeks.

Short-timescale motion of the centroid position of Sgr A* would be expected if a portion of the emission comes from material orbiting about the SMBH. The degree of centroid variability would necessarily depend on the brightness of the orbiting material, the degree to which its emission is nonuniform, and the orbital radius dominating the total flux. We use a simple hot spot model to relate the constraint from the observed short-term position wander of Sgr $A^{*}(\S 2)$ to a constraint on the presence of strong inhomogeneities in the accretion flow onto the SMBH as a function of hot spot luminosity and orbital period ( $(3)$. Finally, concluding remarks are contained in $\S 4$.

\section{OBSERVATIONS OF CENTROID MOTION}

Reid \& Brunthaler (2004) describe the observations and data calibration methods in detail. Briefly, we obtained position data as follows: the VLBA antennas switched between $\mathrm{Sgr} \mathrm{A}^{*}$ and a compact extragalactic source (J1745-2820) every $15 \mathrm{~s}$ in order to provide interferometer phase differences rapidly enough to cancel the effects of short-term atmospheric fluctuations. The stronger source, Sgr A*, was used as the phase reference to calibrate data from the weaker source, J1745-2820.

Astrometric imaging of $\mathrm{Sgr} \mathrm{A}^{*}$ at $7 \mathrm{~mm}$ wavelength is best accomplished with only the five inner-VLBA antennas (FD, KP, $\mathrm{LA}, \mathrm{OV}$, and PT). These antennas produce interferometer baselines with lengths of up to $1500 \mathrm{~km}$, resulting in synthesized beams typically about $2.5 \times 0.7$ mas (FWHM) elongated north-south. Longer baselines (e.g., involving the Washington [BR] and Iowa [NL] state antennas) are not generally useful for precise astrometry, as it is difficult to detect $\mathrm{Sgr} \mathrm{A}^{*}$ with the $8 \mathrm{~s}$ on-source integrations afforded by rapid switching, coupled with low fringe 


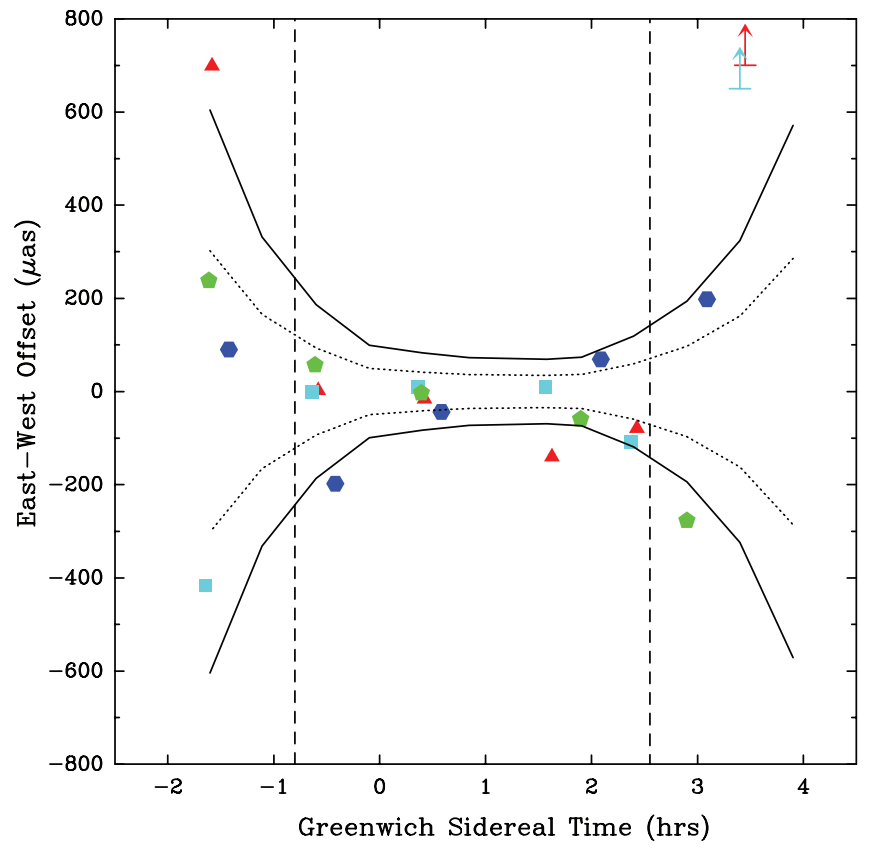

FIG. 1.- Hourly measurements of the centroid position of $\mathrm{Sgr} \mathrm{A}^{*}$ relative to an extragalactic source. Data from 2003 April 5 (red triangles) and 25 (cyan squares) and 2007 April 5 (green pentagons) and 11 (blue hexagons) are plotted. All positions are relative to their daily average position. Dotted and solid lines indicate expected $\approx 1 \sigma$ and $\approx 2 \sigma$ uncertainties, based on Monte Carlo simulations of the effects of uncompensated atmospheric path delays $(0.5 \mathrm{~cm} \mathrm{rms})$ at each antenna. Data outside the vertical dashed lines were excluded from analysis owing to their large expected position errors.

visibilities on long baselines owing to the large, scatter-broadened image of Sgr A* Also, the sources are mutually visible with the inner five antennas for only a short time period for antennas far from the inner ones.

Our most accurate astrometry was obtained with atmospheric path delay calibration using "geodetic" blocks (Reid \& Brunthaler 2004). This involves short periods of observations of quasars with a wide spanned bandwidth and scheduled to deliver a wide range of source elevations. These geodetic blocks were placed before the start, at the middle, and after the end of the Sgr A* observations. Analysis of these data yields estimates of the zenith atmospheric path delay at each antenna accurate to $\sim 0.5-1 \mathrm{~cm}$ (or about 1 wavelength).

\subsection{Position Wander: Hours}

For analysis of short-term wander, we selected only our highest quality data, requiring both high-accuracy atmospheric path delay calibration using geodetic blocks (which was started in 2003) and data from all five inner-VLBA antennas. Data from VLBA programs BR84 on 2003 April 5 and 25 and BR124 on 2007 April 5 and 11 satisfied these requirements.

The position wander of Sgr A* over timescales of hours was determined by dividing the calibrated interferometer data into hourly bins. The data were Fourier transformed to make images, and intensity centroid positions were determined. In practice, we measured the background source, J1745-2820, which had been phase referenced to $\operatorname{Sgr}^{*}$, but we interpret any position changes as owing to changes in Sgr A*. In Figure 1 we show the east-west position offsets as a function of Greenwich sidereal time, after removing an average position for each day's data. The north-south positions are intrinsically less accurate by a factor of about 3 , as the north-south projections of the interferometer baselines are correspondingly shorter than the east-west projections.
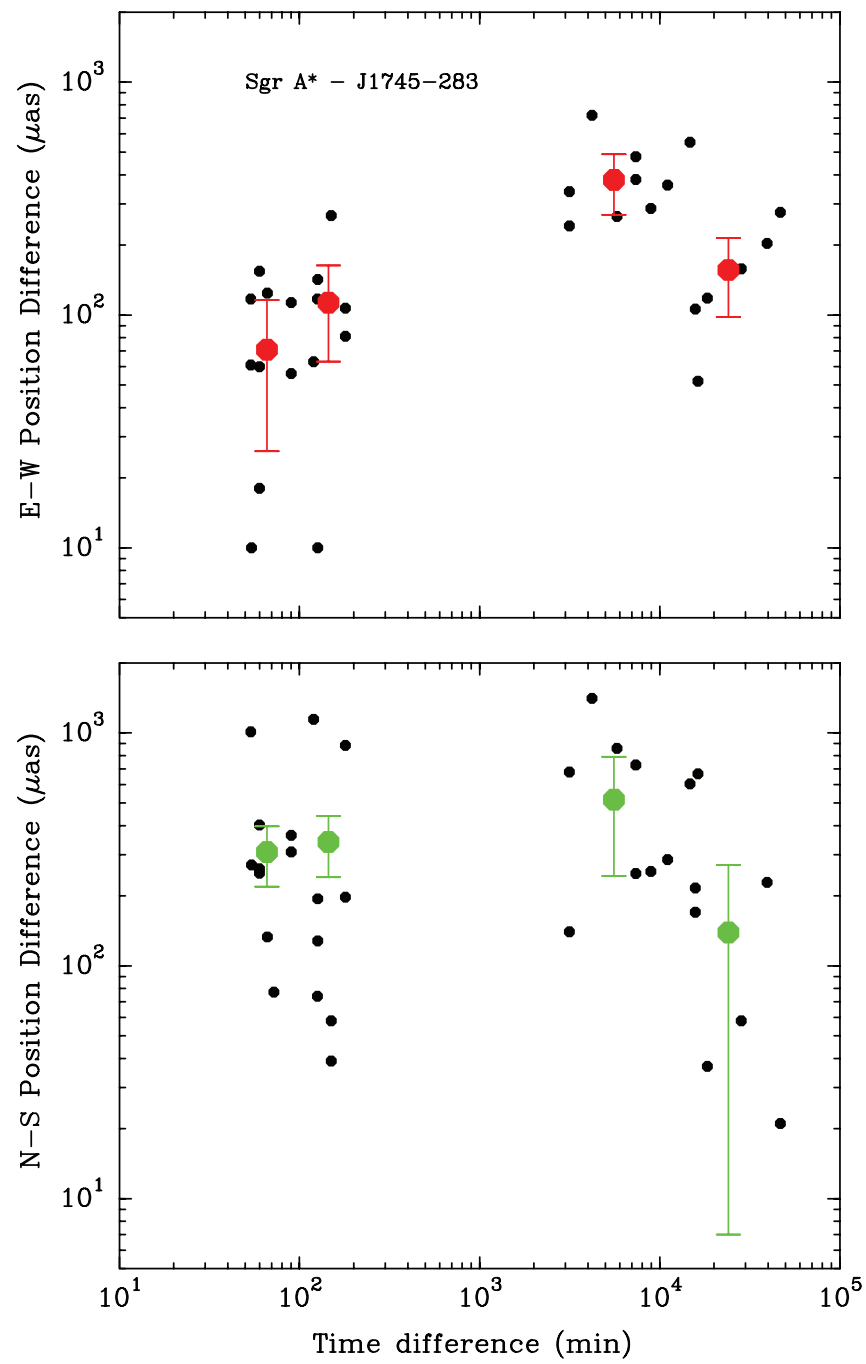

FIG. 2.-Position wander of Sgr A*. Plotted are east-west (top) and northsouth (bottom) position differences as a function of time separation. Also shown are binned average position differences as large points with error bars. These position differences are likely caused by uncompensated atmospheric path delay errors and should be considered as upper limits to the position wander of Sgr A*.

At the start and end of the observing tracks, the sources are at low elevations and susceptible to large interferometer phase shifts caused by uncompensated atmospheric path delays. We estimated the effects of zenith path delay errors with 1000 independent simulations. Each simulation started by randomly selecting zenith path delay errors $(0.5 \mathrm{~cm} \mathrm{rms})$ for each antenna. We then calculated the expected interferometer phase shifts, dependent on timevarying source zenith angles, at half-hour time steps. Position shifts were estimated as the product of the phase shifts (in turns) multiplied by an approximate projected interferometer fringe spacing. Finally, we calculated a weighted position shift for all baselines and all times when the source was above $15^{\circ}$ elevation. The resulting expected $\pm 1 \sigma$ and $\pm 2 \sigma$ position error envelopes are plotted in Figure 1. Based on the observed and simulated increase in position scatter for Greenwich sidereal times before $-0.8 \mathrm{hr}$ and after $+2.6 \mathrm{hr}$, we only use data within this time range for our study of hourly position wander.

We differenced the east-west and north-south positions for all pairs of measurements separated by less than $10^{3}$ minutes; the magnitudes of these position differences are shown on the lefthand side of Figure 2, along with two binned weighted averages. The average difference in position for measurements separated 
by $50-100$ minutes is $71 \pm 45 \mu$ as and for measurements separated by $100-200$ minutes is $113 \pm 50 \mu$ as. Neither average position difference deviates significantly from zero, and the position differences are consistent with systematic errors owing predominantly to mismodeling zenith propagation path delays through the Earth's atmosphere at the $\approx 1 \mathrm{~cm}$ level. Thus, we use these measurements as upper limits for the position wander of the centroid of Sgr A*'s emission.

\subsection{Position Wander: Days to Weeks}

The position wander of Sgr A* on timescales of days to weeks was determined from "daily" position measurements, obtained from $\approx 5 \mathrm{hr}$ of data when source elevations were above $\approx 20^{\circ}$ at most of the five inner-VLBA antennas. These positions were plotted in Figure 3 of Reid \& Brunthaler (2004) after removing

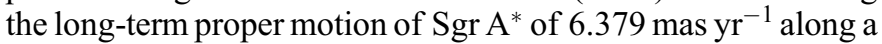
position angle of $209.60^{\circ}$ east of north. We differenced the eastwest and north-south positions for all pairs of measurements separated by less than $10^{5}$ minutes; the magnitudes of these position differences are shown in Figure 2, along with weighted averages for two time bins.

The average difference in east-west position for measurements separated by $\approx 6000$ minutes $(\approx 4$ days) is about $400 \mu$ as. This difference is likely not a property of the emission of Sgr $\mathrm{A}^{*}$; instead it is probably caused by mismodeling large-scale propagation delays through the Earth's atmosphere. Most of the data used in this analysis were collected before we started to measure atmospheric path-lengths in 2003. Without this calibration, residual zenith path length errors of $\sim 5 \mathrm{~cm}$ are typical for the VLBA correlator model at $7 \mathrm{~mm}$ wavelength. Since propagation delays can be correlated with large-scale weather patterns, which have characteristic timescales of several days, one expects to see such a residual signature in our position differences.

\section{CONSTRAINTS ON HOT SPOT MODELS OF Sgr A* VARIABILITY}

Limits on the variability in the centroid position of highresolution images of Sgr $\mathrm{A}^{*}$ imply a constraint on orbiting hot spot models for Sgr A*'s radio variability. This will necessarily be a function of the hot spot orbit and the hot spot-to-disk flux ratio. In this section we derive the expected variability for two simple hot spot models. The first is an optically thin Newtonian hot spot, while the second fully incorporates general relativity and the opacity of a disk constructed such that it reproduces the observed spectrum of Sgr A*.

\subsection{Idealized Newtonian Hot Spot Centroid Variability}

For the idealized Newtonian hot spot we assume that there is no lensing by the black hole, the disk is completely optically thin, and the hot spot is a point (the first two are physically appropriate for spots at large orbital radii). In this case, the hot spot's orbit (which we assume to be circular with radius $r$ ) is simply given by Kepler's law,

$$
x(t)=r \cos (\Omega t+\phi), \quad y(t)=r \cos i \sin (\Omega t+\phi),
$$

where $i$ is the orbital inclination,

$$
\Omega=\sqrt{\frac{G M_{\mathrm{Sgr} \mathrm{A}^{*}}}{r^{3}}}
$$

$M_{\text {Sgr A* }}$ is the mass of Sgr $\mathrm{A}^{*}$, and $\phi$ is an arbitrary phase.
The image centroid, $\boldsymbol{X}_{C}$, is constructed by integrating the source emission over a time $T$, which need not be small in comparison to the orbital period, and thus, the motion of the hot spot will generally be important. Explicitly, if we set the centroid of the disk emission to be at the origin,

$$
\boldsymbol{X}_{C}=\frac{\int_{0}^{T} F_{\text {spot }}(t) \boldsymbol{x}(t) d t}{\int_{0}^{T}\left[F_{\text {disk }}+F_{\text {spot }}(t)\right] d t} .
$$

This will generally be a function of the initial phase of the orbit, $\phi$, and the integration time $T$. If we make the simplifying assumption that the hot spot flux may be treated as roughly constant, then this reduces to

$$
\boldsymbol{X}_{C}=\eta \overline{\boldsymbol{x}}(\phi ; T), \quad \text { where } \quad \eta \equiv \frac{F_{\text {spot }}}{F_{\text {disk }}+F_{\text {spot }}},
$$

and $\overline{\boldsymbol{x}}(\phi ; T)$ is the average spot position over some time $T$ with initial orbital phase ${ }^{6} \phi$. It is straightforward to show that

$$
\begin{gathered}
\bar{x}(\phi ; T)=r \operatorname{sinc}\left(\frac{\Omega T}{2}\right) \cos \phi, \\
\bar{y}(\phi ; T)=r \operatorname{sinc}\left(\frac{\Omega T}{2}\right) \cos i \sin \phi,
\end{gathered}
$$

where a factor of $\Omega T / 2$ has been subsumed into the arbitrary phase $\phi$. This corresponds to a single observation of the position of $\operatorname{Sgr} \mathrm{A}^{*}$.

To study the position wander, we must compare two such measurements separated by some time $\Delta T$. This results in a change in the centroid position of

$$
\begin{aligned}
\left|\Delta \boldsymbol{X}_{C}\right|(\phi ; T, \Delta T)= & \eta|\overline{\boldsymbol{x}}(\phi+\Omega \Delta T ; T)-\overline{\boldsymbol{x}}(\phi ; T)| \\
= & \eta r \operatorname{sinc}\left(\frac{\Omega T}{2}\right) \sin \left(\frac{\Omega \Delta T}{2}\right) \\
& \times \sqrt{\cos ^{2} \phi+\cos ^{2} i \sin ^{2} \phi .}
\end{aligned}
$$

Maximizing the position wander over the arbitrary initial phase simply removes the terms in the radical,

$$
\left|\Delta \boldsymbol{X}_{C, \max }\right|(T, \Delta T)=\eta r \operatorname{sinc}\left(\frac{\Omega T}{2}\right) \sin \left(\frac{\Omega \Delta T}{2}\right) .
$$

While it may be unlikely that any given observation of the position wander will allow detection of the maximum centroid displacement, this quantity places a strict constraint on hot spots. Alternatively, one may wish to compare the observations to the predicted rms centroid variability, which in this case is roughly $70 \%$ of $\left|\Delta \boldsymbol{X}_{C, \max }\right|(T, \Delta T)$.

For fixed $T$ and $\Delta T,\left|\Delta \boldsymbol{X}_{C, \max }\right|$ vanishes when an integral number of periods is commensurate with either the integration time (and thus $\overline{\boldsymbol{x}}(\phi ; T)$ vanishes identically) or the delay between measurements. These are evident in Figure 3, in which the thin blue line shows $\left|\Delta \boldsymbol{X}_{C, \max }\right|$ as a function of orbital period for a number of values of $\eta$ with $T=1 \mathrm{hr}$ and $\Delta T=4 \mathrm{hr}$. However, by choosing many different $T$ and $\Delta T$, it is possible fill in

\footnotetext{
${ }^{6}$ While this assumption may appear to be manifestly unjustified, for an optically thick hot spot in the Rayleigh-Jeans limit, the final expression is formally accurate! The reason is that the relativistic beaming of the hot spot emission is precisely countered in this instance by the combination of the Doppler boosting and time-of-flight delays (Broderick \& Loeb 2005).
} 

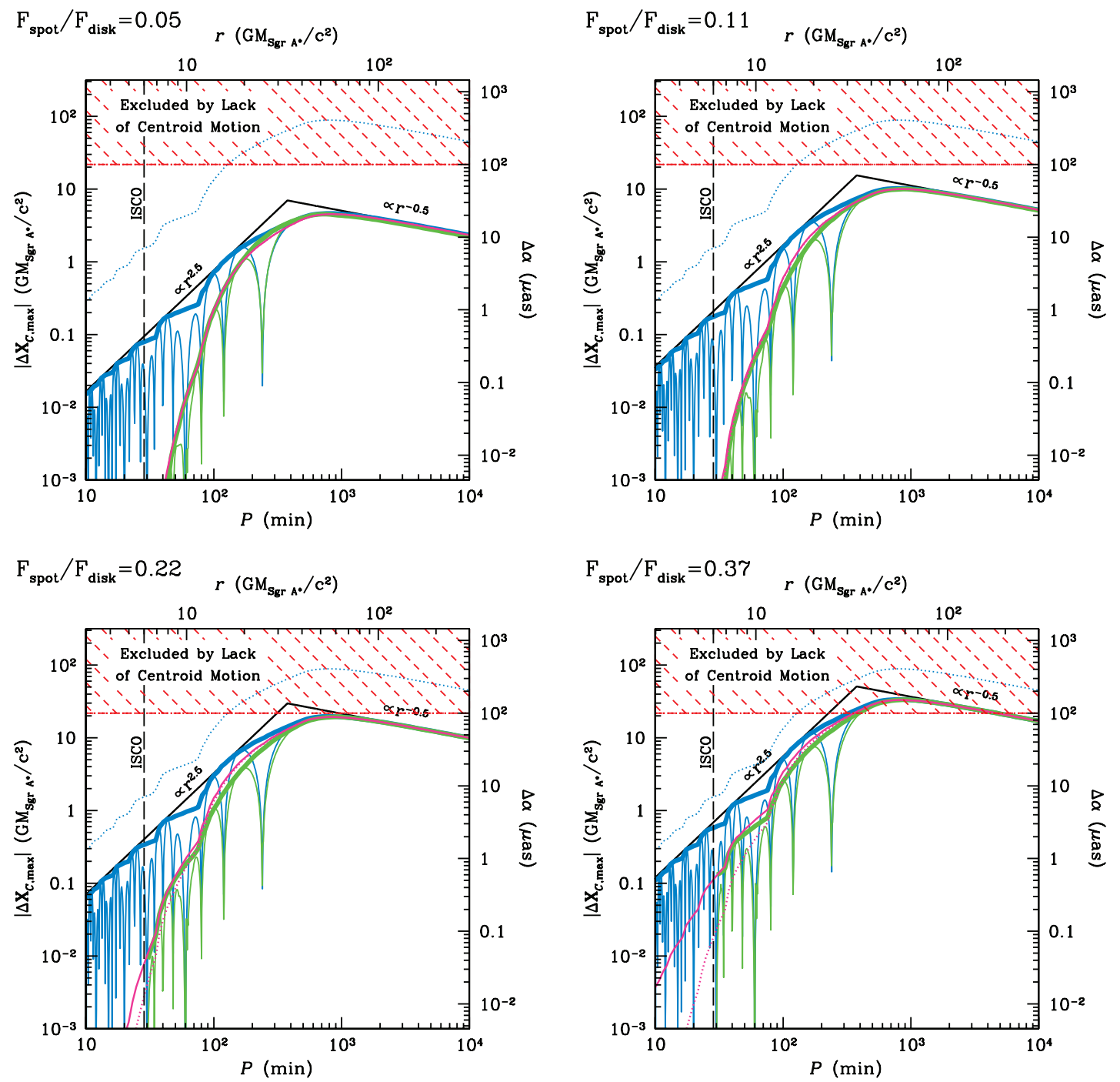

FIG. 3.- Maximum observed physical displacement of the centroid as a function of the orbital period for the idealized Newtonian case (blue lines) and the fully

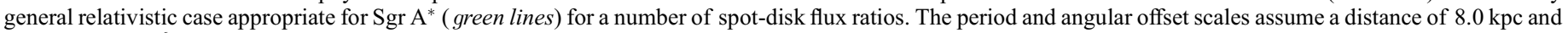
$M_{\mathrm{Sgr} \mathrm{A}^{*}}=4 \times 10^{6} M_{\odot}$. The thin blue and green lines show the variation associated with observations integrated over $T=1 \mathrm{hr}$ and separated by $\Delta T=4 \mathrm{hr}$. The thick blue and green lines show the envelope for all observation strategies with $T \geq 1 \mathrm{hr}$ and $\Delta T \leq 4$. In all cases, the thin dotted blue line shows the maximum deviation

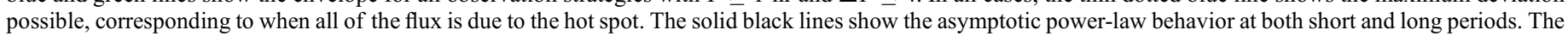

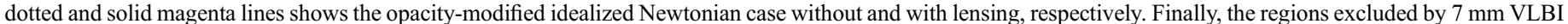

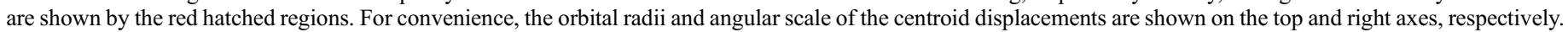

the nulls associated with each individual choice. There will be constraints on what values of $T$ and $\Delta T$ are possible, e.g., due to sensitivity and imaging requirements (see $\S 2$ ). The upper envelope of the observed centroid displacement for $1 \mathrm{hr} \leq T \leq 4 \mathrm{hr}$ and $1 \mathrm{hr} \leq \Delta T \leq 4 \mathrm{hr}$ is shown in Figure 3 by the thick, solid blue line. For reference we also show, with dotted blue lines, the maximum deviation possible, corresponding to the case when all of the flux is due to the hot spot.

Generally, the observed displacement has a maximum near orbital periods of roughly $\pi(T \Delta T)^{1 / 2}$. That such a maximum must exist can be inferred from the facts that (1) at large orbital radii, the Keplerian velocity decreases, and thus, beyond some distance the hot spot will move sufficiently slow as to produce no detectable change in it's position over the time $\Delta T$; and (2) at small orbital radii, the intrinsic variability in the spot position is smaller and the hot spot makes many complete orbits in the integration time $T$, and thus, the variable portion of the centroid position is dominated by a small fraction of the integrated flux, yielding again a small observable change in the centroid position. However, we may also show this explicitly by considering the asymptotic expansions of equation (7). For small $\Omega$ (large period $P$ ), the sinc term is roughly unity and

$$
\left|\Delta \boldsymbol{X}_{C, \max }\right|(T, \Delta T)=\eta r \frac{\Omega \Delta T}{2} \propto r^{-1 / 2} .
$$

In contrast, for large $\Omega,\left|\Delta \boldsymbol{X}_{C, \max }\right|(T, \Delta T)$ is strongly oscillatory. Nevertheless, it is bounded from above by the sinc term, and thus,

$$
\left|\Delta \boldsymbol{X}_{C, \max }\right|(T, \Delta T) \leq \frac{2 \eta r}{\Omega T} \propto r^{5 / 2} .
$$

Setting these limiting expressions equal to each other gives the desired condition that a maximum observed displacement occurs near $\Omega \simeq 2 /(T \Delta T)^{1 / 2}$. Note that this is true for the envelope obtained by varying $T$ and $\Delta T$ in the prescribed ranges as well and serves as a simple estimate of the sensitivity of these types of measurements. 


\subsection{Fully Relativistic, Hot Spot in an Optically Thick Disk}

We now consider a more realistic model in which a hot spot is embedded in an accretion disk, including the relativistic beaming, Doppler boosting, strong gravitational lensing, and the opacity of the disk and hot spot. This is necessarily a more complicated model, and thus, we address it numerically via the ray-tracing, radiative transfer code described in Broderick \& Loeb (2006; to which we direct the reader for more information, the model only being summarized below).

Because of its ability to shield the hot spot from view, the structure of the background disk is of particular importance. In the absence of an unambiguous prediction from existing accretion flow theory, we have modeled it as a self-similar radiatively inefficient accretion flow (RIAF), following Yuan et al. (2003). Specifically, the accretion flow is characterized by a Keplerian velocity distribution, a population of thermal electrons with density and temperature

$$
n_{e, \mathrm{th}}=n_{e, \mathrm{th}}^{0} r^{-1.1} e^{-z^{2} / 2 \rho^{2}}, \quad T_{e}=T_{e}^{0} r^{-0.84},
$$

respectively, a population of nonthermal electrons

$$
n_{e, \mathrm{nth}}=n_{e, \mathrm{nth}}^{0} r^{-2.9} e^{-z^{2} / 2 \rho^{2}},
$$

spectral index $\alpha_{\text {disk }}=1.25$ (defined as $S \propto \nu^{-\alpha}$ ), and a toroidal magnetic field in approximate $(\beta=10)$ equipartition with the ions (which produce the majority of the pressure), i.e.,

$$
\frac{B^{2}}{8 \pi}=\beta^{-1} n_{e, \mathrm{th}} \frac{m_{p} c^{2}}{6 r} .
$$

In all the expressions, $r, \rho$, and $z$ are measured in units of $G M_{\mathrm{Sgr} \mathrm{A}} \mathrm{A}^{*} c^{2}$. The power laws are taken from Yuan et al. (2003), and the three coefficients $\left(n_{e, \text { th }}^{0}, T_{e}^{0}\right.$, and $\left.n_{e, \text { nth }}^{0}\right)$ are set by fitting the radio, submillimeter, and near-infrared spectrum of Sgr $\mathrm{A}^{*}$. While this is not a unique model for the accretion flow around Sgr A*, it is representative of the general class of RIAFs, and we expect that our results will be quite generic.

The hot spot is modeled by a spherical (in its frame) Gaussian overdensity of nonthermal electrons, with a radial scale of $1.5 G M_{\mathrm{Sgr} \mathrm{A}^{*}} / c^{2}$. The primary distinction between the systems shown in the panels of Figure 3 is the size of the photosphere of the hot spot $(\delta r)$, larger hot spots necessarily being more luminous.

The radiative transfer is assumed to be dominated by synchrotron emission and absorption. We follow Jones \& O'Dell (1977) for the power-law electron distributions in the accretion flow and the hot spot, cutting the electron distributions off below Lorentz factors of $10^{2}$, roughly in agreement with the assumptions in Yuan et al. (2003). We treat the radiative transfer of the thermal component of the accretion flow in a similar fashion as that discussed in Yuan et al. (2003) appropriately altered to account for the relativistic nature of the bulk motion (see, e.g., Broderick \& Blandford 2004 for more detail on how this may be done).

The centroid displacements were computed by (1) generating a sequence of images associated with an entire orbit, (2) determining the instantaneous image centroid for each, (3) inserting these into equation (3) to determine the time-integrated centroid position for a given $T$, and finally (4) given a $\Delta T$, varying the orbital phase until the maximum $\left|\Delta \boldsymbol{X}_{C, \max }\right|(T, \Delta T)$ was obtained. The result for $T=1 \mathrm{hr}$ and $\Delta T=4 \mathrm{hr}$ is shown by the thin green line in Figure 3 and exhibits many of the features found in the idealized Newtonian case. This procedure was repeated a num- ber of times for randomly chosen $T \in[1 \mathrm{hr}, 4 \mathrm{hr}]$ and $\Delta T \in$ [ $1 \mathrm{hr}, 4 \mathrm{hr}]$ to produce the upper envelope of the centroid displacements and is shown by the thick green line in Figure 3.

The primary effect is the suppression of the centroid variability due to the opacity of the background accretion flow. This suppression becomes substantial at radii near the location of the photosphere, which is approximately $12.5 G M_{\mathrm{Sgr} \mathrm{A}^{*} / c^{2}}$ at $7 \mathrm{~mm}$ wavelength. In contrast, at large orbital radii the idealized Newtonian model fits quite well. This interpretation is further supported by the fact that the idealized Newtonian model fits quite well once it has been modified to include opacity. Specifically, as a rough approximation, we reduce the hot spot flux by a factor of $e^{-\tau}$, where

$$
\tau=\left(\frac{r+\delta r}{12.5}\right)^{-2.99} \propto r n_{e, \text { nth }} B^{\alpha_{\text {disk }}+3 / 2}
$$

is the optical depth associated with the nonthermal electrons in the accretion flow. This is shown by the magenta lines in Figure 3. While we should also include the thermal electron component, at $7 \mathrm{~mm}$ wavelength the nonthermal electrons appear to dominate the opacity.

The free parameter $\delta r$ is associated with the finite extent of the hot spot. That is, it is possible for the spot to be visible even if the hot spot center is inside of the accretion flow photosphere. It is for this reason that the centroid variability is more similar to the idealized Newtonian value for high-luminosity spots than that for low-luminosity spots. Setting $\delta r$ using the hot spot photosphere radius alone gives the dotted magenta line, which underestimates the centroid variability considerably at small radii for large (bright) hot spots. This is due to the failure of the idealized Newtonian calculation to account for the strong lensing of large spots in small orbits (with $r_{\text {spot }} \simeq r$ ). However, simply employing a larger $\delta r$ (64\% larger at the largest disk-to-hot spot flux ratio we consider) results in a substantially improved fit.

\section{DISCUSSION}

Possible reasons for position wander include intrinsic variations in the position of the emitting plasma (e.g., variations in the accretion flow or perhaps in a jet) or extrinsic processes such as refractive interstellar scattering. Sgr $\mathrm{A}^{*}$ is observed to be diffractively scattered to a size of $\theta_{\mathrm{sc}} \sim 0.5(\lambda / 0.7 \mathrm{~cm})^{2}$ mas, where $\lambda$ is the observing wavelength. Flux density fluctuations are modest and decrease in strength with increasing wavelength; thus, strong refractive scintillations are not indicated (Gwinn et al. 1991). Any refractive position wander should be $\ll \theta_{\mathrm{sc}}$ and should occur on timescales $>\theta_{\mathrm{sc}} D / v$, where $D$ is the distance and $v$ is the transverse velocity of the scattering "screen" relative to the observer (Romani et al. 1986). For $D \approx R_{0} \approx 8 \mathrm{kpc}$ (Reid 1993) and $v \sim 100 \mathrm{~km} \mathrm{~s}^{-1}$, characteristic of material in the inner $\sim 100 \mathrm{pc}$ of the Galaxy where large scattering sizes are observed, the refractive timescale is $>10^{3}$ hours. Thus, we would not expect a significant contribution to the short-term wander of $\mathrm{Sgr} \mathrm{A}^{*}$ from refractive scattering. For comparison, Gwinn et al. (1988), using very long baseline interferometry (VLBI) observations of the $\mathrm{Sgr} \mathrm{B} 2(\mathrm{~N}) \mathrm{H}_{2} \mathrm{O}$ masers near the Galactic center, find a wander limit of $<18 \mu$ as over timescales of months for maser spots, which are diffractively scattered to a comparable size (at $22 \mathrm{GHz}$ ) as Sgr A* (at $43 \mathrm{GHz}$ ). Of course, our results provide an observation limit to any refractive position wander.

Since extrinsic sources of position wander (scattering) are unlikely to be dominant, we now discuss the implications for intrinsic wander from variations in brightness within an accretion disk given in $\S 3$. Our observations of the lack of short-term wander of 
the centroid position of $\mathrm{Sgr} \mathrm{A}^{*}$ presented in $\S 2.1$ give an upper limit of $\approx 100 \mu$ as for timescales of $\approx 1-4 \mathrm{hr}$. This translates to an upper limit on the wander versus orbital period plots in Figure 3 as indicated by the horizontal red line and hatched region. (In the very unlikely event that the accretion disk inclination is both near $90^{\circ}$ and oriented nearly north-south on the sky, we would need to use our north-south limits, which are a factor of 3 weaker.) Our east-west limit is below the dotted blue line in Figure 3, which is for hot spot flux density dominating over disk (or possible jet) emission, for orbital periods exceeding 120 minutes (corresponding to orbital radii larger than $15 G M_{\mathrm{Sgr} \mathrm{A}}{ }^{*} / c^{2}$ for $M_{\text {Sgr A }}{ }^{*}=4 \times$ $\left.10^{6} M_{\odot}\right)$. For cases in which the hot spot flux density is weaker than that of the disk, somewhat longer periods are allowed. For example, for $F_{\text {spot }} / F_{\text {disk }}=0.37$, orbital periods longer than $5 \mathrm{hr}$ are excluded.

In practice, the limits placed by current $7 \mathrm{~mm}$ VLBI are significantly weaker. The limited sensitivity to hot spots on compact orbits is primarily due to two reasons: (1) "long" integration times ( $T \gtrsim 1 \mathrm{hr}$ ) average much of the short-time variability out, and (2) the opacity of the accretion flow itself makes it difficult to view hot spots on compact orbits at $7 \mathrm{~mm}$. The integration time is limited by the sensitivity issues and the small number of antennas yielding interferometer baselines $<1500 \mathrm{~km}$ afforded by the current VLBA; higher bandwidth recording in the future should help alleviate this problem. The optical depth is a property of Sgr A* itself and can only be addressed by observations at shorter wavelengths. However, even in the absence of an optically thick accretion flow, it is not possible to increase the cen- troid variability by more than an order of magnitude due to the intrinsically small orbital radii, as seen by comparing the blue and green limits in the bottom left panel of Figure 3.

Nevertheless, high-resolution astrometry is reaching sensitivities and resolutions sufficient to begin to test the hot spot model for bright Sgr A* flares. Unfortunately, the typical fractional variability at $7 \mathrm{~mm}$ during our observations was roughly $\pm 20 \%$, implying that significant improvement in positional accuracy will be required to constrain such events. Since the observed centroid wander is consistent with systematic errors, owing predominantly to centimeter-scale errors in the modeling of the atmospheric path delays, substantially increasing accuracy will require better calibration techniques. However, for the somewhat rare instances in which the spot is substantially brighter (Zhao et al. 2001), the VLBA at 7 , or possibly 3 , mm wavelength appears poised to provide significant limits on the existence and morphology of inhomogeneities in the accretion flow surrounding Sgr A*.

Ultimately, observations at $\sim 1 \mathrm{~mm}$ wavelength with VLBI techniques or at infrared wavelengths with an instrument like GRAVITY (Gillessen et al. 2006) may be necessary to image the region within $\sim 3$ Schwarzschild radii on the short timescales needed to test the hot spot model.

A.B. is supported by the Priority Programme 1177 of the Deutsche Forschungsgemeinschaft.

Facilities: VLBA

\section{REFERENCES}

Bower, G. C., Falcke, H., Herrnstein, R. M., Zhao, J.-H., Goss, W. M., \& Backer, D. C. 2004, Science, 304, 704

Broderick, A., \& Blandford, R. 2004, MNRAS, 349, 994

Broderick, A. E., \& Loeb, A. 2005, MNRAS, 363, 353 . 2006, MNRAS, 367, 905

Dehnen, W., \& Binney, J. J. 1998, MNRAS, 298, 387

Ghez, A. M., et al. 2003, ApJ, 586, L127

Gillessen, S., et al. 2006, Proc. SPIE, 6268, 33

Gwinn, C. R., Danen, R. M., Tran, T. Kh., Middleditch, J., \& Ozernoy, L. M. 1991, ApJ, 381, L43

Gwinn, C. R., Moran, J. M., Reid, M. J., \& Schneps, M. H. 1988, ApJ, 330, 817
Jones, T. W., \& O’Dell, S. L. 1977, ApJ, 214, 522

Menten, K. M., Reid, M. J., Eckart, A., \& Genzel, R. 1997, ApJ, 475, L111 Reid, M. J. 1993, ARA\&A, 31, 345

Reid, M. J., \& Brunthaler, A. 2004, ApJ, 616, 872

Reid, M. J., Menten, K. M., Genzel, R., Ott, T., Schödel, R., \& Eckart, A. 2003, ApJ, 587, 208

Romani, R. W., Narayan, R., \& Blandford, R. 1986, MNRAS, 220, 19

Schödel, R., et al. 2002, Nature, 419, 694

Yuan, F., Quataert, E., \& Narayan, R. 2003, ApJ, 598, 301

Zhao, J.-H., Bower, G. C., \& Goss, W. M. 2001, ApJ, 547, L29 